\title{
Bilateral torque asymmetry analysis of lower limbs of active and insufficiently active older adults
}

\author{
MILENA RAZUK ${ }^{1,2}$ | JULIANA C. LORETE1 | LEONARDO A. VIEIRA'1,2 | NATALIA M. RINALDI1,2
}

1 Strength and Conditioning Laboratory (LAFEC) of Center of Physical Education and Sports, Department of Sports, Federal University of Espírito Santo, Vitória, ES, Brazil. ${ }_{2}$ Biomechanical Movement Analysis Laboratory (BIO.MOV), Department of Sports, Federal University of Espírito Santo, Vitória, ES, Brazil.

Correspondenceto: Milena Razuk, Center of Physical Education and Sports, Department of Sports, Federal University of Espírito Santo, Brazil. Av. Fernando Ferrari, 514 - Goiabeiras, Vitória - ES, 29075-910. Tel: (27) 4009-2620.

email: milena.razuk@gmail.com

https://doi.org/10.20338/bjmb.v14i2.162

HIGHLIGHTS
- Older adults showed similar behavior for
bilateral asymmetry, regardless of the level of
physical activity.
-The physical training program did not produce
a reduction in bilateral torque asymmetry.
- The bilateral torque asymmetry was greater
for the ankle and hip joints in the flexion and
extension movements, respectively.
ABBREVIATIONS
ATV Active group
BA $\quad$ bilateral asymmetry
BAMP bilateral asymmetry mean power
BAPT bilateral asymmetry of peak torque
EGS $\quad$ Exercise Guidance Service
IATV Insufficiently active group
MP $\quad$ mean power
Nm N Newton meter
PT $\quad$ peak torque
W $\quad$ Watt

HIGHLIGHTS

- Older adults showed similar behavior for bilateral asymmetry, regardless of the level of physical activity.

- The physical training program did not produce a reduction in bilateral torque asymmetry.

bilateral torque asymmetry was greater extension movements, respectively.

PUBLICATION DATA

Received 07022020

Accepted 19042020

Published 01072020
BACKGROUND: Physical activity is an important tool for positively impacting physical, cognitive, and social aspects and is linked to the capacity to develop muscle strength and power. However, no studies have observed the effects of physical activity on the bilateral torque asymmetry at different movement velocities in older adults. AIM: This study investigated the effects of different angular velocities on bilateral torque asymmetry for hip, knee, and ankle joints in active (EGS program) and insufficiently active older adults.

METHOD: Forty-four older adults were distributed into two groups: active and insufficiently active. An isokinetic dynamometer was used. The tests consisted of maximal concentric contractions of hip, knee, and ankle flexors and extensors at velocities of $60 \%$ and $120 \%$ s. The variables were asymmetry of peak torque and of mean power.

RESULTS: Results showed greater asymmetry in hip extension and ankle flexion for peak torque and mean power, as well as increased asymmetry in flexion movement at the higher angular velocity $(120 \% \mathrm{~s})$ compared with the lower angular velocity $\left(60^{\circ} / \mathrm{s}\right)$ for the mean power. Furthermore, there were no differences in asymmetry between groups.

CONCLUSION: The conclusion of the present study was that older adults showed similar behavior for bilateral torque asymmetry, regardless of the level of physical activity. The level of physical activity did not produce a reduction in bilateral torque asymmetry in the active group.

KEYWORDS: Asymmetric index | Muscle function | Angular velocity

\section{INTRODUCTION}

According to the World Health Organization, population aging is a global phenomenon, resulting from factors that include a drop in fertility rates and increase in life expectancy. ${ }^{1}$ Currently, there are approximately 962 million people over 60 in the world, corresponding to $13 \%$ of the global population. This population group grows at a rate of around $3 \%$ per year, and it is estimated that by 2050 there will be approximately 2 billion older adults in the world, of which $80 \%$ will be living in developing countries. ${ }^{1}$ In Brazil, the aging of the population is occurring even faster, with a growth rate of over $4 \%$ per year, ${ }^{2}$ so that by 2025 , Brazil will be the sixth country in the world in terms of number of older adults. ${ }^{1}$ The population aged 60 and above increased from 14.2 million in 2000 to 19.6 million in 2010, and will reach 41.5 million in 2030 and 66.5 million in 2050, corresponding to $30 \%$ of the Brazilian population. ${ }^{2}$

With aging, structural and functional deterioration may occur in most physiological

\begin{tabular}{l|l|l|l|l}
\hline Razuk et al. & 2020 & VOL.14 & N.2 & https://doi.org/10.20338/bjmb.v14i2.162
\end{tabular}


systems, which can cumulatively impact the activities of daily living, functional independence, level of physical activity, and quality of life of older adults. ${ }^{3}$ Similarly, older adults show a $20-40 \%$ reduction in muscle strength compared to younger adults. ${ }^{3}$ Muscle strength and power reach their maximum values around the third decade of life, demonstrating a decline with the progression of age and intensifying after 60 years of age. ${ }^{4}$ The decline in muscle function as a consequence of the aging process can be explained by the decrease in the number of motor units and also the decrease and atrophy in the number of muscle fibers, especially type II, which are important for the production of force and explosive actions. ${ }^{5}$ Thus, the decline in muscle strength is aggravated by contractile deceleration and decreased torque during aging. ${ }^{5}$

In this context, the peak of torque (i.e., maximal voluntary force output) is a neuromuscular variable directly related to muscle strength and is the most appropriate indicator of maximal performance of a muscle group. ${ }^{5}$ The torque produced by the musculature depends on the type of contraction, angular position of the joint and velocity of contraction. Torque is an important force variable for the performance of daily life activities and impairment in this variable can increase the risk of falling. ${ }^{6}$ Studies have found lower knee and ankle joint torques in faller older adults compared to non-fallers. ${ }^{6}$

Symmetrical actions are necessary for many activities of daily living. ${ }^{7}$ However, most people have a preferred lower or upper limb to perform these activities, ${ }^{8}$ which can generate bilateral asymmetry $(B A)$ in muscle strength between lower limbs and between different muscle groups. ${ }^{9}$ Thus, BA characterizes a difference in performance between the right and left lower limbs, ${ }^{10}$ as well as between injured and uninjured lower limbs, ${ }^{11}$ and between dominant and non-dominant lower limbs. ${ }^{10}$ Results from one study, ${ }^{12}$ showed that older adults with $20 \%$ or more of isometric knee extensor strength asymmetry demonstrated a more asymmetrical and variable gait pattern and walked $19 \%$ more slowly than their more symmetrical peers. This study led to the hypothesis that strength asymmetry may be associated with physical dysfunction in older adults and that the weaker limb may limit gait performance. Likewise, other studies infer that lower limb asymmetry may be a predictor of falls ${ }^{6}$ in older adults with a diagnosis of Parkinson's disease. ${ }^{13}$

In contrast, studies have found that resistance training (i.e., free weight exercises for muscle strengthening) benefits older adults, as training provides a sharp increase in muscle mass, strength, and power, ${ }^{14}$ improves body composition ${ }^{15}$ and bone mineral density, decreases the risk of falls and following injuries, ${ }^{14}$ increases cognitive function, and generally improves quality of life by minimizing aging and muscle degeneration processes. ${ }^{16}$ In addition, according to the results of a case study, ${ }^{17}$ older adults rehabilitated after hip fracture through participation in a multimodal resistance training protocol, demonstrated gains in muscle strength and better symmetry between the lower limbs.

Physical activity is an important tool for positively impacting physical, cognitive, and social aspects ${ }^{18}$ and is linked to the protection of functional capacity at different ages, with the ability to develop muscle strength and power ${ }^{19}$. Among the types of physical activities, programs that aim to simultaneously develop strength, balance, and flexibility, known as multicomponent programs, seem to be linked to improvements in functionality in older adults. ${ }^{20}$ Thus, one of the main programs aimed at promoting health through the orientation of physical activity is the Exercise Guidance Service (EGS), created by the Municipal Secretariat of Vitória/ES in 1985, which serves people from various age groups. 
The main activities developed by the EGS program, classified as multicomponent exercises, are stretching, strengthening, balance, and aerobic, with recreational activities for socialization at the end of the sessions, twice a week, lasting approximately 60 minutes. The physical training program provides exercises that are equally challenging for both sides of the body.

Despite being a stable program, operating for approximately 30 years, there is little evidence on the health benefits of EGS in the population, more specifically on the deleterious effects in the musculoskeletal system caused by aging. Moreover, it is important to understand the influence of the practice of multicomponent exercises, such as EGS, on performance in bilateral torque asymmetry of older adults, as this type of exercise is frequently recommended for health. ${ }^{21}$ The current study investigated the effects of different angular velocities on bilateral torque asymmetry for hip, knee, and ankle joints in active (EGS program) and insufficiently active older adults. The hypothesis of this study was that the insufficiently active group would present higher values of bilateral asymmetry of peak torque (BAPT) and of mean power (BAMP) for hip, knee, and ankle joints than the active group. The argument that supports this first hypothesis is that the mechanical loading and muscular forces experienced by an individual as a result of regular physical activity would be similar between limbs and neuromuscular adaptations that occur in response to these activities might be expected to be comparable. A physically active person may still present asymmetry but have greater strength and power in both strong and weak limbs than a less active person..$^{22}$ In addition, we expected that the $60 \%$ angular velocity would present higher BAPT than the $120 \%$ s angular velocity due to the higher peak torque (PT) for the lower velocity. The argument that supports this second hypothesis is that power asymmetry might be manifested most notably at fast contraction velocities, possibly due to the greater demand for motor unit activation, recruitment of type II muscle fibers, and limitations in nerve conduction velocity, excitation-contraction coupling, and contractile function. ${ }^{23}$ Finally, we expected that the $120 \%$ s angular velocity would present higher BAMP than the $60 \%$ angular velocity due to the higher mean power (MP) at the higher velocity. The argument that supports this third hypothesis is that lower velocity allows a longer time to develop maximal torque and may be more sensitive to identify strength. ${ }^{24}$

\section{METHODS}

\section{Participants}

Forty-four older adults participated in this study, distributed into two groups: 1) Active group (ATV - 22 participants, $65.2 \pm 4.2$ years | $66.5 \pm 9.6 \mathrm{~kg} \mid 1.60 \pm 0.06 \mathrm{~m}$ ) used as the experimental group, composed of older adults who had regularly practiced multicomponent exercises offered by the Exercise Guidance Service (EGS) for more than three months, twice a week, in sessions with 60 minutes of duration; 2) Insufficiently active group (IATV - 22 participants, $66.7 \pm 4.5$ years | $68.2 \pm 10.5 \mathrm{~kg} \mid 1.60 \pm 0.05 \mathrm{~m}$ ), composed of older people who had not practiced physical activity in the previous three months. Participants were excluded from the study if they had neurological and musculoskeletal disorders that prevented them from performing motor tasks, and cognitive impairment. All participants provided consent prior to data collection. The study was approved by the Research Committee of the Federal University of Espírito Santo (2.061.608). 


\section{Apparatus and procedures}

Data collection was performed on two days. On the first day, anamnesis and anthropometric evaluations were carried out in EGS units (Vitória/ES). The physical activity level was assessed through the modified Baecke Questionnaire for older adults, ${ }^{25}$ which investigates daily sports and leisure activities. The Mini-Mental State Examination validated for the Brazilian population - Mini Mental26 was used to assess the cognitive function of older adults (active and insufficiently active). The authors ${ }^{26}$ propose 20 points for illiterate individuals; 25 points for people with schooling from 1 to 4 years; 26.5 for 5 to 8 years; 28 for 9 to 11 years, and 29 for over 11 years. On the second day, muscle function was evaluated in the Strength and Conditioning Laboratory (LAFEC), located in the Physical Education and Sports Center of the Federal University of Espírito Santo (CEFD/UFES).

The participants performed a 5 minute warm-up on a cycle ergometer with a selfselected load. After this, these subjects were positioned and stabilized on the BIODEX System 4 Pro isokinetic dynamometer according to the manufacturer's recommendations. Hip flexion and extension, knee flexion and extension, and plantar flexion and ankle dorsiflexion movements were assessed, with the order randomized for each participant. The participants performed 5 submaximal repetitions at each angular velocity to familiarize themselves with the test. The assessment consisted of concentric isokinetic contractions with predetermined angular velocities and repetitions of $60 \%$ s (five repetitions) and $120 \% \mathrm{~s}$ (ten repetitions). Participants were verbally encouraged to develop maximum strength during the test with a rest interval of 60 seconds between different angular velocity assessments. Measurements were collected bilaterally, always starting with the dominant limb (the preferred one to kick a ball). The calibration and gravity correction procedures were performed according to the manufacturer's recommendations. Muscle assessment tasks were collected in separate blocks and counterbalanced within the group. Trials were completely randomized within each block.

\section{Data analysis}

The dependent variables used to assess torque asymmetry were bilateral asymmetry of mean power (BAMP) and bilateral asymmetry of peak torque (BAPT) at angular velocities of $60 \%$ and $120 \%$ for flexion and extension movements.

The mean power is the result of the work done divided by time, expressed in Watts (W). The angular velocity is directly proportional to the power (i.e., the higher the angular velocity, the greater the power; the lower the angular velocity, the lower the power).

The peak torque represents the point of greatest torque in the range of movement; torque or moment of force represents the result of the force applied at a point multiplied by the distance from the point of application of that force to the center of rotation of the axis of movement (i.e., $T=F x d$, measured in Newton meters $(\mathrm{Nm})$ ). Torque and angular velocity of movement are inversely proportional quantities, i.e., the lower the angular velocity performed, the greater the torque, the higher the angular velocity, the lower the torque.

Finally, the symmetric index, considering the average of each participant in each type of movement (absolute values), was calculated between the dominant and nondominant limbs. However, we were interested only in the magnitude of symmetry, and did not consider the positivity or negativity of the values. ${ }^{13}$ 
Symmetric index $=\left(\frac{\text { value of dominant limb }- \text { value of non dominant limb }}{\text { value of dominant limb }+ \text { value of non dominant limb }}\right) \times 100 \%$

\section{Statistical analysis}

After testing the normality and homogeneity of variance assumptions, repeated measures MANOVAs three-way with group (insufficiently active and active), angular velocities $(60 \%$ and $120 \% \mathrm{~s})$, and joints (hip, knee, ankle), were performed for each dependent variable: BAMP and BAPT. Independent sample t tests were performed to compare clinical characteristics (Mini-Mental and Baecke Questionnaire). When necessary, post hoc tests with Bonferroni adjustment were performed. Analyses were performed using SPSS software and the level of significance was kept at $p<0.05$.

\section{RESULTS}

In relation to the level of physical activity, measured by the Baecke Questionnaire, the active EGS group presented a higher level of physical activity compared to the insufficiently active group (ATV: $13.9 \pm 4.3$ points | IATV: $3.8 \pm 4.3$ points, $p \leq 0.001$ ). In relation to the Mini-Mental score, the statistical analysis revealed no differences between the older active group and older insufficiently active group (ATV: $27.87 \pm 1.67$ points | IATV: $27.80 \pm 1.40$ points, $p=0.858$ ).

Table 1 depicts the mean values of the BAMP for insufficiently active and active groups at both angular velocities $(60 \% \mathrm{~s}$ and $120 \% \mathrm{~s})$ for flexion and extension movements of the hip, knee, and ankle joints. The MANOVA three-way revealed no mean effect of group (Wilks' Lambda $=0.966 ; F_{2,41}=0.726 ; p=0.49$ ), but a mean effect of angular velocity (Wilks' Lambda $=0.869 ; F_{2,41}=3.088 ; p=0.05$ ), and a mean effect of joint (Wilks' Lambda $\left.=0.507 ; F_{2,41}=9.476 ; p<0.001\right)$. The Univariate tests for angular velocity indicated an effect of flexion movement $\left(F_{1,42}=13.839 ; p<0.001\right.$, but no effect of extension movement $\left(F_{1,42}=6.573 ; p=0.002\right)$. The BAMP value for flexion movement was higher at $120 \% \mathrm{~s}(76.6 \pm 11.8)$ than at $60 \%(66.7 \pm 11.2)$. The univariate tests for joint indicated an effect of flexion $\left(F_{1,42}=286.97 ; p<0.001\right)$, and extension movements $\left(F_{1,42}=\right.$ 308.21; $p$ <0.001). Post hoc tests for joint flexion movement were larger in the ankle joint than in knee and hip joints. Finally, post hoc tests for extension movement were higher in the hip joint than knee and ankle joints. 
Brazilian Journal of Motor Behavior

Table 1 - Mean and standard deviation of bilateral asymmetry of mean power (BAMP) in flexion and extension movements of the hip, knee, and ankle joints for insufficiently active and active older groups.

\begin{tabular}{|c|c|c|c|c|}
\hline Group & Velocity & Joint & $\begin{array}{c}\text { Flexion } \\
\text { Mean } \pm \text { Standard } \\
\text { deviation }\end{array}$ & $\begin{array}{c}\text { Extension } \\
\text { Mean } \pm \text { Standard } \\
\text { deviation }\end{array}$ \\
\hline \multirow{6}{*}{$\begin{array}{l}\text { Insufficiently } \\
\text { Active }\end{array}$} & \multirow{3}{*}{$60 \% \mathrm{~s}$} & Hip & $5.91 \pm 4.86$ & $15.37 \pm 20.91$ \\
\hline & & Knee & $11.91 \pm 9.55$ & $6.50 \pm 4.76$ \\
\hline & & Ankle & $18.94 \pm 24.25$ & $9.50 \pm 10.62$ \\
\hline & \multirow{3}{*}{$120 \% / \mathrm{s}$} & Hip & $7.88 \pm 5.33$ & $13.02 \pm 13.33$ \\
\hline & & Knee & $12.57 \pm 11.51$ & $6.91 \pm 5.00$ \\
\hline & & Ankle & $22.90 \pm 23.69$ & $9.86 \pm 13.01$ \\
\hline \multirow{6}{*}{ Active } & \multirow{3}{*}{$60 \% / \mathrm{s}$} & Hip & $6.12 \pm 6.48$ & $14.38 \pm 16.21$ \\
\hline & & Knee & $6.58 \pm 5.43$ & $7.20 \pm 5.30$ \\
\hline & & Ankle & $17.24 \pm 16.94$ & $8.60 \pm 5.81$ \\
\hline & \multirow{3}{*}{$120 \% \mathrm{~s}$} & Hip & $5.20 \pm 6.11$ & $13.38 \pm 11.38$ \\
\hline & & Knee & $8.79 \pm 7.88$ & $5.82 \pm 3.68$ \\
\hline & & Ankle & $19.26 \pm 16.85$ & $9.44 \pm 8.89$ \\
\hline
\end{tabular}

Table 2 depicts the mean values of the BAPT for insufficiently active and active groups in both angular velocities $(60 \%$ and $120 \% \mathrm{~s})$ for flexion and extension movements of the hip, knee, and ankle joints. The MANOVA three-way revealed no mean effect of group (Wilks' Lambda $=0.979 ; F_{2,41}=0.438 ; p=0.648$ ), or of velocity (Wilks' Lambda $=$ $0.96 ; F_{2,41}=0.853 ; p=0.434$ ), but a mean effect of joint (Wilks' Lambda $=0.454 ; F_{2,41}=$ $11.73 ; p<0.001)$. The univariate tests for joint indicated an effect for flexion $\left(F_{1,42}=13.394\right.$; $p<0.001)$ and extension movements $\left(F_{1,42}=4.546 ; p=0.013\right)$. Post hoc tests for joint flexion movement were higher in the ankle joint than in knee and hip joints. Finally, post hoc tests for extension movement were higher in the hip joint than in knee and ankle joints.

Table 2 -Mean and standard deviation of bilateral asymmetry of peak torque (BAPT) in flexion and extension movements of the hip, knee, and ankle joints for insufficiently active and active older groups

\begin{tabular}{|c|c|c|c|c|}
\hline Group & Velocity & Joint & $\begin{array}{c}\text { Flexion } \\
\text { Mean } \pm \text { Standard } \\
\text { deviation }\end{array}$ & $\begin{array}{c}\text { Extension } \\
\text { Mean } \pm \text { Standard } \\
\text { deviation }\end{array}$ \\
\hline \multirow{6}{*}{$\begin{array}{l}\text { Insufficiently } \\
\text { Active }\end{array}$} & \multirow{3}{*}{$60 \% \mathrm{~s}$} & Hip & $4.53 \pm 3.31$ & $10.87 \pm 11.60$ \\
\hline & & Knee & $10.55 \pm 6.85$ & $5.98 \pm 5.16$ \\
\hline & & Ankle & $13.47 \pm 18.53$ & $6.66 \pm 7.74$ \\
\hline & \multirow{3}{*}{$120 \% / s$} & Hip & $5.59 \pm 4.94$ & $7.65 \pm 7.98$ \\
\hline & & Knee & $8.63 \pm 7.65$ & $6.11 \pm 4.74$ \\
\hline & & Ankle & $11.11 \pm 13.54$ & $8.25 \pm 8.38$ \\
\hline \multirow{6}{*}{ Active } & \multirow{3}{*}{$60 \% / \mathrm{s}$} & Hip & $3.77 \pm 2.01$ & $9.22 \pm 8.97$ \\
\hline & & Knee & $4.89 \pm 3.06$ & $5.01 \pm 3.35$ \\
\hline & & Ankle & $14.29 \pm 11.10$ & $9.25 \pm 5.47$ \\
\hline & \multirow{3}{*}{$120 \%$ s } & Hip & $5.07 \pm 4.27$ & $8.95 \pm 8.25$ \\
\hline & & Knee & $5.14 \pm 4.11$ & $4.60 \pm 2.62$ \\
\hline & & Ankle & $12.78 \pm 9.02$ & $6.82 \pm 6.88$ \\
\hline
\end{tabular}

\section{DISCUSSION}

The purpose of this study was to investigate the effects of different angular velocities on bilateral torque asymmetry for hip, knee, and ankle joints in active (EGS program) and insufficiently active older adults. The main results of the study showed 
higher mean power asymmetry and higher peak torque asymmetry in the ankle joint for flexion movement and hip joint for extension movement. Furthermore, the mean power asymmetry at the velocity of $120 \% \mathrm{~s}$ was greater than at the velocity of $60 \%$ s for flexion movement, which partially confirmed our hypothesis. Finally, the results showed no difference between the active and insufficiently active groups for the variables mean power asymmetry and peak torque asymmetry, which was contradictory to our hypothesis.

In the present study an increase in bilateral torque asymmetry was observed for the hip extension movement and ankle flexion movement for both peak torque and mean power. These results may be related to the aging process, which promotes a deficit in skeletal muscle function, mainly concentric strength and muscle power of the lower limbs. ${ }^{27}$ Thus, analogous to the decline in muscle function, the aging process promotes changes in postural strategies, with greater dependence on the hip strategy over the ankle strategy for postural control. ${ }^{28}$ These changes seem to occur due to muscle weakness, slowness, and impairment in the organization to activate muscle synergies and limitations in the ability to adapt movements for balance in response to changing task and environmental demands. ${ }^{28}$ In the same context of environmental demand, researchers ${ }^{29}$ demonstrated a decrease in area under unstable conditions (foam) with eyes open and closed in the active older adults (group of multicomponent exercises). The study revealed that active older adults presented improved performance of postural control in challenging conditions of somatosensory manipulation associated with manipulation of the visual system. Thus, multicomponent exercises are effective for improving postural control performance in older adults. These findings infer that body position and weight distribution between the feet interact to stabilize upright posture.

Surprisingly, results from one study 6 showed that the decline in strength and power in older adults is related to the rate of falls in this population. Many situations in daily life rely on symmetrical postural control, such as responding to balance perturbation or walking, to avoid falls. These factors are related to muscle weakness, aging, and altered patterns of muscle activation (coordination) which indicates a decline in physical and/or cognitive abilities that affect postural control and, consequently, safe mobility 6 . In the same study ${ }^{6}$, the power loss associated with increased velocity in the plantar flexors of the older adults was also verified and corroborates the results found in the present study in which the relationship between power and angular velocity may be a factor for the increase in the power asymmetry identified at the velocity of $120 \% \mathrm{~s}$ compared to the velocity of $60 \%$ s. Similarly, other research ${ }^{12}$ conducted with older adults found that power asymmetry was higher at higher velocities. A possible explanation for this result may be due to the recruitment of type II muscle fibers and the reduction in conduction velocity in nerve impulses in older adults. It is important to note that these authors ${ }^{12}$ found a power reduction in the weak leg while power in the strong leg increased proportionally with increasing speed. This fact may be related to the limb preference to perform daily tasks, resulting in performance asymmetry between the right and left lower limbs. ${ }^{7}$

Although studies have demonstrated the benefits of exercise, reducing the effects of aging and, consequently, improving the quality of life, ${ }^{14,16}$ the present study found no difference in bilateral asymmetry of mean power and peak torque between the active and insufficiently active groups. In contrast, the active group in the EGS program participated in a multimodal program of collective physical/body activities (gymnastics, stretching, yoga, circuit, dance, walking and other activities), which provide better performance of balance and muscle power. ${ }^{30}$ However, the program does not include activities focused on strength 
training specifically for lower limb joints. Thereby, it can be concluded that the bilateral asymmetry developed during life does not decrease in active older adults and was similar to the insufficiently active group. Other researchers ${ }^{17}$ showed improvement in bilateral asymmetry of older adults after the application of a high intensity multimodal resistance training protocol that resulted in muscle strength gain and decreased asymmetry between the lower limbs according to the specificity of the training protocol.

The present study presents some limitations. First, we did not carry out a pre- and post-training investigation of the modalities offered by EGS to elucidate possible changes caused by each of the practices. Second, the EGS program aims to improve social aspects (e.g., leisure and socialization), thus, it is difficult to control training parameters (e.g., overload and intensity) which could provide more accurate data on alterations and adaptations in the participants. Third, it may be possible that a more natural, ecologically valid situation (compared to the protocol used in the present study), with a reduced force requirement task (and increased sensory information based adjustments), such as postural control, would reveal bilateral asymmetry improvements from the exercise program.

\section{CONCLUSION}

The conclusion of the present study was that older adults showed similar behavior for bilateral asymmetry, regardless of the level of physical activity. The level of physical activity did not produce a reduction in bilateral torque asymmetry in the active group. This may be due to the protocol of activities offered by the EGS which, despite including various body practices does not include a specific training protocol for muscle strength gain capable of reducing bilateral asymmetry, and thus further improving the quality of life of the older adults. Furthermore, the explosion variable (mean power) was sensitive to the angular velocity increase $(120 \% \mathrm{~s})$ for flexion movement, showing greater bilateral asymmetry. Finally, the bilateral asymmetry for both variables was greater for the ankle joint in the flexion movements and greater for the hip joint in the extension movements.

\section{REFERENCES}

1. Organização Mundial da Saúde (OMS). Relatório mundial de envelhecimento e saúde. Genebra, 2015. https://sbgg.org.br/wp-content/uploads/2015/10/OMS-

ENVELHECIMENTO-2015-port.pdf Accessed 01 January 2020.

2. IBGE. Instituto Brasileiro de Geografia e Estatística. Relações entre as alterações históricas na dinâmica demográfica brasileira e os impactos decorrentes do processo de envelhecimento da população. Rio de Janeiro: IBGE, 2016.

https://biblioteca.ibge.gov.br/pt/bibliotecacatalogo?view=detalhes\&id=298579.Accessed 01 January 2010.

3. American College of Sports Medicine (ACSM). American College of Sports Medicine position stand. Exercise and physical activity for older adults. Med Sci Sport Exer. 2009;41(7):1510-30.

4. Deschenes MR. Effects of aging on muscle fibre type and size. Sports Med. 2004;34(12):809-824. doi: https://doi.org/10.2165/00007256-200434120-00002. 
5. Fazolin MAFG. Efeito das sessões de familiarização sobre o pico de torque e taxa de desenvolvimento de torque = comparações entre jovens, meia idade e idosos. 2013. $70 \mathrm{f}$. Dissertação (mestrado) - Universidade Estadual de Campinas Faculdade de Educação Física, Campinas, SP.Available in: http://repositorio.unicamp.br/jspui/handle/REPOSIP/275091. Accessed 01 January 2020.

6. Crozara LF, Morcelli MH, Hallal CZ et al. Effect of age and fall status on lower extremity muscle activation and joint torque and power in physically active women. Isokinet Exerc Sci. 2016;24(1):67-77. doi: https://doi.org/10.3233/IES-150602.

7. Simon AM, Ferris DP. Lower limb force production and bilateral force asymmetries are based on sense of effort. Exp Brain Res. 2008;187:129-138. doi: https://doi.org/10.1007/s00221-008-1288-x.

8. Yoshioka S, Nagano A, Hay DC, Fukashiro $S$. The effect of bilateral asymmetry of muscle strength on jumping height of the countermovement jump: a computer simulation study. $J$ Sports Sci. 2010;28(2):209-218. doi: https://doi.org/10.1080/02640410903428566.

9. Fousekis $K$, Tsepis $E$, Vagenas $G$. Lower limb strength in professional soccer players: profile, asymmetry, and training age. J Sports Sci Med. 2010;9(3):364-373.

10. Jones PA, Bampouras TM. A comparison of isokinetic and functional methods of assessing bilateral strength imbalance. J Strength Cond Res. 2010;24(6):1553-1558. doi: https://doi.org/10.1519/JSC.0b013e3181dc4392.

11. Paterno MV, Ford KR, Myer GD, Heyl R, Hewett TE. Limb asymmetries in landing and jumping 2 Years following anterior cruciate ligament reconstruction. Clin J Sport Med. 2007;17(4):258-262. doi: https://doi.org/10.1097/JSM.0b013e31804c77ea.

12. Laroche DP, Cook S, Mackala K. Strength asymmetry increased gait asymmetry and variability in older women. Med Sci Sports Exerc. 2012;44(11):2172-81. doi: http://doi.org/10.1249/MSS.0b013e31825e1d31.

13. Beretta VS, Barbieri FA, Simieli Let al. Can Postural Control Asymmetry Predict Falls in People With Parkinson's Disease? Motor Control. 2018;22(4), 449-461. doi: http://dx.doi.org/ 10.1123/mc.2017-0033.

14. Schott N, Johnen B, Holfelder, B. Effects of free weights and machine training on muscular strength in high-functioning older adults. Exp Gerontol. 2019;122:15-24. doi: https://doi.org/10.1016/j.exger.2019.03.012.

15. Cavalvante EF, Ribeiro AS, do Nascimento MA et al. Effects of different resistance training frequencies on fat in overweight/obese older women. Int J Sport Med. 2019;39(7): 527-534. doi: https://doi.org/10.1055/a-0599-6555.

16. McKinnon NB, Connelly DM, Rice CL, Hunter SW, Doherty TJ. Neuromuscular contributions to the age-related reduction in muscle power: mechanisms and potential role of high velocity power training. Ageing Res Rev. 2017;35:147-154. doi: https://doi.org/10.1016/j.arr.2016.09.003.

17. Briggs RA, Houck JR, LaStayo PC, Fritz JM, Drummond MJ, Marcus RL. High-intensity multimodal resistance training improves muscle function, symmetry during a sit-to-stand 
task, and physical function following hip fracture. J Nutr Health Aging. 2018;20(3):431-328. doi: https://doi.org/10.1007/s12603-017-0977-1.

18. Da Silva MV, Aguillar IN, Rocco DD. Avaliação isocinética de pico de torque e relação agonista/antagonista em jogadores de futebol da categoria sub-23. Anais do Encontro Nacional de Pós Graduação. 2018;2(1):357-362.

19. Granacher U, Muehlbaue T, Zahner L, Gollhofer A, Kressig RW. Comparison of Traditional and Recent Approaches in the Promotion of Balance and Strength in Older Adults. Sports Medicine. 2011;41(5):377-400.

20. Sousa JL. Implementação e avaliação da eficácia de um programa de exercício físico multicomponente, orientado para a funcionalidade, em pessoas idosas. 2016. Tese de Doutorado. Universidade de Santiago de Compostela.

21. Piercy KL, Troiano RP, Ballard RM et al. The Physical Activity Guidelines for Americans. JAMA. 2018;320(19):2020-2028.

22. Laroche DP, Villa MR, Bond CW, Cook SB. Knee extensor power asymmetry is unrelated to functional mobility of older adults. Exp Gerontol. 2017;98:5461.https://doi.org/10.1016/j.exger.2017.08.008.

23. Babault N, Pousson M, Michaut A, Ballay Y, Hoecke JV. EMG activity and voluntary activation during knee-extensor concentric torque generation. Eur J Appl Physiol. 2002;86(6):541-547.https://doi.org/10.1007/s00421-002-0579-3.

24. Grabiner MD, Owings TM, Pavol MJ. Lower extremity strength plays only a small role in determining the maximum recoverable lean angle in older adults. $J$ Gerontol A BiolSciMed Sci. 2005;60(11):1447-1450.

25. Voorrips LE, Ravelli AC, Petra C, Dongelmans A, Deurenberg P, Van Starven WA. A physical activity questionnaire for the elderly. Medicine and Science in Sports and Exercise. 1991;29:117-121.

26. Brucki SM, Nitrini R, Caramelli P, Bertolucci PH, Okamato IH. Suggestions for utilization of the mini-mental state examination in Brazil. Arq Neuropsiquiatr. 2003;61(3B):777-781.

27. Raj IS, Bird SR, Shield AJ. Aging and the force-velocity relationship of muscles. Exp Gerontol. 2010;2:81-90.

28. Horak FB. Postural orientation and equilibrium: what do we need to know about neural control of balance to prevent falls? Age Ageing. 2006;2:7-11.

29. Hue, O. Effects of a physical activity program on postural stability in older people. Aging Clin Exp Res. 2004;16(5):356-362.

30. Vieira LA. Análise do desempenho e da influência da força e potência muscular dos membros inferiores no equilíbrio postural de idosos ativos e sedentários. 2019. f.154Dissertação (mestrado) - Universidade Federal do Espírito Santo, Vitória. 
Citation: Razuk M, Lorete JC, Vieira LA, Rinaldi NM. Bilateral torque asymmetry analysis of lower limb of active and insufficiently active older adults. BJMB. 2020: 14(2): 62-72.

Editors: Dr Fabio Augusto Barbieri - São Paulo State University (UNESP), Bauru, SP, Brazil; Dr José Angelo Barela São Paulo State University (UNESP), Rio Claro, SP, Brazil.

Copyright:@2020 Razuk, Lorete, Vieira and Rinaldi and BJMB. This is an open-access article distributed under the terms of the Creative Commons Attribution-NonCommercial-No Derivatives 4.0 International License which permits unrestricted use, distribution, and reproduction in any medium, provided the original author and source are credited. Funding: There was no funding for this study.

Competing interests: The authors have declared that no competing interests exist. DOI: https://doi.org/10.20338/bjmb.v14i2.162 